\title{
MACROPARASITE COMMUNITY AND ASYMMETRY OF THE YELLOW EEL ANGUILLA ANGUILLA IN SALSES-LEUCATE LAGOON, SOUTHERN FRANCE
}

\author{
G. FAZIO (1), R. LECOMTE-FINIGER (2), J. BARTRINA (2), H. MONÉ (1), P. SASAL (1)
}

(1) Laboratoire de Parasitologie Fonctionnelle et Évolutive, UMR 5555 CNRS, Université de Perpignan, 58, avenue Paul Alduy, 66860 Perpignan Cedex, France. E-mail: fazio@univ-perp.fr

(2) École Pratique des Hautes Études, UMR 8046 CNRS, Université de Perpignan, 58, avenue Paul Alduy, 66860 Perpignan Cedex, France.

Reçu le 6 octobre 2004

Accepté le 10 novembre 2005

Received October 6, 2004 Accepted November 10, 2005

\begin{abstract}
European eel parasites, in particular invasive species, are suspected to have a strong influence on the population dynamics of their host. The aim of this work was to study the relationship between parasitic fauna of yellow eels caught at Salses-Leucate lagoon and the fluctuating asymmetry (FA) of some functional bilateral traits: pectoral fins, eyes and otoliths. Epidemiological distance matrices and FA distance matrices were calculated on the basis of the Euclidian distances between each pair of eel and were then compared with a Mantel test in order to establish if there was a relationship between parasitism and FA. Our results revealed different morphological characteristics between eels caught at different dates; only otoliths were found asymmetric. The parasite richness was of 10 species, 1 Nematoda, 1 Acanthocephala, 1 Monogenea and 7 Digenea. We failed to find a significant relationship between asymmetry and parasitism, except for parasites found in the stomach. We discussed the results in the light of the eel life cycle, the specificity of parasite - eel systems and the impact of parasites on the host physiology.
\end{abstract}

Key-words: Parasites, eels, fluctuating asymmetry, distance matrices, SalsesLeucate lagoon.

\section{COMMUNAUTÉ DES MACROPARASITES ET ASYMÉTRIE CHEZ L'ANGUILLE JAUNE ANGUILLA ANGUILLA DANS LA LAGUNE DE SALSES-LEUCATE, SUD DE LA FRANCE}

\section{RÉSUMÉ}

Les parasites de l'anguille européenne, en particulier les espèces invasives, sont supposés avoir une grande influence sur la dynamique des populations de leur hôte. Ce travail se propose d'étudier la relation entre la faune parasitaire d'anguilles jaunes prélevées dans la lagune de Salses-Leucate et l'asymétrie fluctuante $(F A)$ de traits bilatéraux fonctionnels : les nageoires pectorales, les yeux et les otolithes. Des matrices de distance épidémiologique ainsi que des matrices de distance de FA ont été calculées sur la base des distances euclidiennes entre anguilles prises deux à deux et ont ensuite été comparées par un test de Mantel afin d'établir s'il existait une relation entre elles. Nos 
résultats ont montré des caractéristiques morphologiques différentes entre les anguilles selon la date de capture; seuls les otolithes présentent une asymétrie. Nous avons trouvé une richesse parasitaire de 10 espèces, 1 Nematoda, 1 Acanthocephala, 1 Monogenea et 7 Digenea. II n'existe pas de relation entre l'asymétrie et le parasitisme, à l'exception des parasites trouvés dans l'estomac. Nous discutons les résultats à la lumière du cycle biologique de l'anguille, de la spécificité des systèmes parasites - anguille et de l'impact des parasites sur la physiologie de leur hôte.

Mots-clés : Parasites, anguilles, asymétrie fluctuante, matrices de distance, lagune de Salses-Leucate.

\section{INTRODUCTION}

Preserving the European eel, Anguilla anguilla, is of great concern due to its extensive decline (MORIARTY, 1987; BRUSLÉ, 1990; ELIE et al., 1994; CASTELNAUD and ROCHARD, 1994; WIRTH and BERNATCHEZ, 2003). A number of factors have been suggested as causes of the decline in the recruitment and in the abundance of the European eel. Habitat loss or fragmentation due to the blockage of upstream passages, mortalities of silver eels during downstream migration, overexploitation and effect of contaminants on survival and reproduction are the most frequently evoked causes of eel decline (BRUSLÉ, 1994, for a review). Parasites are becoming a major concern in conservation biology because of their ability to evolve rapidly (DASZAK et al., 2000) and are now recognized to play an important role not only in aquaculture but also in natural systems (ALTIZER et al., 2003). The epidemiology of parasites, the dynamic of their introduction in the case of invasive species and their consequences in terms of host behaviour, growth and reproductive success need thus to be studied in order to protect endangered species.

Fluctuating asymmetry (FA) is the most used measurement of developmental instability; it is a macroscopic measure of the difference of development between left and right from perfect bilateral symmetry (VAN VALEN, 1962), caused by environmental or biotic stresses (VAN VALEN, 1962; MARKOV, 1995; MØLLER and SWADDLE, 1997). Parasites have been found to be associated with a deviance of symmetry in the development (MØLLER, 1992; POLAK, 1993; REIMCHEN, 1997, THOMAS et al., 1998, BROWN and BROWN, 2002 but see also CLARKE, 1998 and PALMER, 1999). This may be explained by (i) a direct stress caused by the metabolic cost of the parasite on its host (MØLLER, 1992; POLAK, 1993), (ii) a variation of the abundance of the parasite related to more or less stressful environmental conditions for hosts (KOSKIVAARA and VALTONEN, 1992), (iii) a relationship between the genetic incapacity to interfere with developmental errors and the susceptibility to parasite (MØLLER, 1996). Several studies have tested a relationship between FA and parasitism. However, results were not obvious especially when the analyses were conducted on several bilateral traits. These confusing results were mainly due to (i) a low concordance between FA of different traits on a same individual (NOSIL and REIMCHEN, 2001; PÉLABON et al., 2004) and (ii) the fact that not all the measured characters were concerned when a relationship between parasitism and asymmetry was found (BERG et al., 1997; ALIBERT et al., 2002). We chose to work on functional bilateral traits such as (i) pectoral fins, used for locomotion and equilibrium, (ii) eyes, used for perception of the environment and indicator of the silvering process (PANKHURST, 1982), (iii) otoliths, used for maintenance of stability and perception of depth pressure, gravity, angular movements and sound waves (BLACKER, 1974). Moreover, we may hypothesize that if an asymmetry on one trait may not be important on the life of the fish, a sum of asymmetry could interfere with the life history traits of an individual (such as reproductive success and survival). As a consequence, beside the study of simple relationships between a class of parasites species (Digenea, Nematoda, Ancanthocephala, Monogenea) or the micro habitat in which the parasite lives (gills, stomach, intestine or swimbladder) and the 
asymmetry of a bilateral trait, we decided to calculate an index of asymmetry based on all the measured asymmetric traits. We confronted it to a global index of parasitism based on all the present parasites in each fish studied. We hypothesized that this new approach using all the measured asymmetric traits and all the parasites species should be a more powerful tool (not only in terms of statistical power) to detect, if it exists, a relationship between parasitism and asymmetry. aimed to:

The present study focused on yellow eels caught in Salses-Leucate lagoon and

- make an inventory of the parasitic fauna to evaluate the parasite pressure on the fish living in this site,

- examine the association between FA and parasitism and discuss whether parasites should be considered as causes or consequences of asymmetry if a relationship exists.

\section{MATERIALS AND METHODS}

\section{Sample collection, morphological and epidemiological analyses}

Eighty yellow eels were collected by professional fishermen at Salses-Leucate lagoon (42.8 ${ }^{\circ} \mathrm{N} 3.0^{\circ} \mathrm{E}$, Pyrénées-Orientales, France) in March 2002 (15 fishes), October 2002 (13 fishes), December 2002 (14 fishes) and March 2003 (38 fishes). The eels were brought back to the laboratory alive and maintained in freshwater until dissection. Each fish was measured (standard length, SL) to the nearest $\mathrm{mm}$ and instantly killed by beheading. Morphological measurements were taken to the nearest $0.1 \mathrm{~mm}$ : maximum length of the right and left pectoral fins (LFr and LFI) and horizontal and vertical diameter of the right and left eyes (HDEr, VDEr, HDEl and VDEI). Each trait was measured twice, right and left measurements within a replicate being made during the same measuring session. All measurements were made by a single person to minimize observer variations. The ocular index (OI), which is a measure of eye area to length of the eel (PANKHURST, 1982) was calculated as follows: OI $=\left[((\mathrm{HDE}+\mathrm{VDE}) / 4)^{2} \times \pi / \mathrm{SL}\right] \times 100$. The parasites of the swimbladder were counted during the dissection. Later on, one side of each gill and the digestive tract were examined under a binocular microscope. All the adult parasites were recovered and some of them were mounted under a cover slide for further identification. Species identification was done when possible and classical epidemiological parameters (prevalence, mean intensity and abundance) were calculated according to BUSH et al. (1997).

\section{Otolith analysis}

Sagital otoliths were isolated and measurements were automatically taken with a computer-enhanced video image-analysis system, which also includes the Visilog 5 TM software (Noesis): maximum right and left length (LOr and LOI), maximum right and left width (WOr and WOI) and right and left perimeter (POr and POI). A complete automatic analysis (picture binarisation and measurements) was performed twice on 30 random otoliths pairs. Then, otoliths were embedded in resin and ground (X600 and X1000 grit). After using an EDTA solution, otoliths were stained with toluidine blue to visualise annual rings. The age estimation was made by counting the winter rings (MORIARTY, 1983; LECOMTE-FINIGER, 1985).

\section{Repeatability, measurement error and evaluation of asymmetry}

The repeatability of measurements was assessed as the Pearson correlation coefficient between replicates. Measurements of trait size were highly repeatable $(r>0.95$, $P<0.0001$ for all traits). 
We tested if differences between left and right sides were due to measurement error or reflected true asymmetry. A one-way ANOVA was performed on replicate $(I-r)$ measurements (DAVID et al., 1999). The F-ratio of this test measures true asymmetry relative to measurement error and is usually considered as significant if $F>5$ and $P<0.001$. F-ratios were high and very significant for $L O\left(F_{29,30}=1.84 E+15, P=0.00\right)$, WO $\left(F_{29,30}=1.85 E+15, P=0.00\right)$, and $P O\left(F_{29,30}=1.30 E+15, P=0.00\right)$. However, $F-$ ratios were not significant for $\operatorname{LF}\left(F_{79,80}=4.32, P=0.009\right), \operatorname{HDE}\left(F_{79,80}=0.94, P=0.56\right)$ and $\operatorname{VDE}\left(\mathrm{F}_{79,80}=2.18, \mathrm{P}=0.02\right)$, indicating that these traits were not asymmetric. Consequently, morphological results were given only for the left side for these last three traits, which were thus not incorporated in the further analyses. Analyses were conducted exclusively on otoliths asymmetry.

Fluctuating asymmetry (FA) of length (LO), width (WO) and perimeter (PO) of the otoliths was calculated as the absolute value of the difference between the left and the right size of the character divided by the half sum of both sides (PALMER and STROBECK, 1986).

\section{Statistical analysis}

Kruskal-Wallis non-parametric tests were performed between dates for morphological and otolithometrical characteristics. A Dunn's test (multiple comparison post test) was later performed if $\mathrm{P}<0.05$ to identify significant pair-wise differences. A linear regression was performed between FA values of LO and WO. In order to study the relationships between asymmetry and parasitism, we calculated "epidemiological distances" considering the individual number of parasite of each species as coordinates (SASAL et al., 2004) and "asymmetrical distances" between eels. As it was done for parasites species by SASAL et al. (2004), the different FA values of each eel were considered as the coordinates of an eel in a multi-dimensional space, which allowed us to calculate Euclidian distances between each pair of eel and then distance matrices (UPGMA method). We calculated four FA distance matrices (LO, WO, PO, all characters) and seven epidemiological distance matrices (four with the parasite classes, one considering all parasite species and two with the different niches in which they live). Only two niches (intestine and stomach) were used to calculate distance matrices as gills and swimbladder were parasitized by only one class of parasite, i.e. distance matrices were the same as those calculated with monogeneans and nematodes respectively. Each FA distance matrice was then compared to each epidemiological distance matrice with a Mantel test (1 000 iterations) (MANTEL, 1967).

\section{RESULTS}

\section{Morphological and otolithometrical characteristics}

Table I shows the morphological and otolithometrical characteristics of the 80 yellow eels sampled at Salses-Leucate lagoon between March 02 and March 03. The analysis was not conducted on all the otoliths because some of them were broken during the dissection or unreadable. We tested if the morphological and otolithometrical characteristics of the samples were homogeneous between successive sampling dates and from year to year (Table II). Kruskal-Wallis tests revealed that significant differences existed between dates. Moreover, Dunn's tests showed that the number of significant differences was not homogeneous between dates. Most differences were found between March 02 and October 02, eels caught in March 02 having the smallest values.

Table III shows the FA values for each of the three bilateral traits studied. We tested if there was a relationship between the FA of LO and the FA of WO. No significant relationship existed $(P>0.05)$. We tested if $F A$ of each trait was homogeneous between successive dates and from year to year. No significant differences existed $(P>0.05)$, except for the FA of LO $(11<\mathrm{n}<34 ; \mathrm{H}=9.8$; $\mathrm{P}=0.02)$ : eels caught in December 02 were less asymmetric than eels caught in March $03(\mathrm{P}<0.05)$. 
Table I

Morphological and otolithometrical characteristics of the 80 yellow eels sampled at Salses-Leucate lagoon. SL, standard length; LF, length of the pectoral fins; HDE, horizontal diameter of the eyes; VDE, vertical diameter of the eyes; OI, ocular index; S.D., standard deviation; (min-max), minimal and maximal values of parameters; I, left; r, right. The number of dissected fishes and otoliths may differ due to lost or broken otoliths during dissections.

Tableau I

Caractéristiques morphologiques et otolithométriques des 80 anguilles jaunes échantillonnées à la lagune de Salses-Leucate. SL, longueur standard; LF, longueur des nageoires pectorales; HDE, diamètre horizontal des yeux ; VDE, diamètre vertical des yeux; OI, indice oculaire ; S.D., écart-type ; (min-max), valeurs minimale et maximale des paramètres ; l, gauche ; r, droit. Les différences entre le nombre de poissons disséqués et le nombre d'otolithes sont dues à la perte ou la cassure de certains otolithes pendant les dissections.

\begin{tabular}{|c|c|c|c|c|c|c|}
\hline \multicolumn{2}{|l|}{ Samples } & $\begin{array}{l}\text { March } \\
02\end{array}$ & $\begin{array}{l}\text { October } \\
02\end{array}$ & $\begin{array}{l}\text { December } \\
02\end{array}$ & $\begin{array}{l}\text { March } \\
03\end{array}$ & All \\
\hline \multicolumn{2}{|l|}{$\mathrm{N}$ eels } & 15 & 13 & 14 & 38 & 80 \\
\hline \multicolumn{2}{|l|}{$\begin{array}{l}\text { Age mean } \pm \text { S.D. } \\
(\text { min-max) in years }\end{array}$} & $\begin{array}{l}3.5 \pm 1.3 \\
(2-6)\end{array}$ & $\begin{array}{c}4.5 \pm 0.8 \\
(3.5-6)\end{array}$ & $\begin{array}{l}4.5 \pm 1.4 \\
(2-8)\end{array}$ & $\begin{array}{l}4.5 \pm 0.8 \\
(3-6)\end{array}$ & $\begin{array}{l}4.0 \pm 1.1 \\
(2-8)\end{array}$ \\
\hline \multicolumn{2}{|l|}{$\begin{array}{l}\text { SL mean } \pm \text { S.D. } \\
(\text { min-max) in } m m\end{array}$} & $\begin{array}{l}282 \pm 25 \\
(250-335)\end{array}$ & $\begin{array}{l}339 \pm 38 \\
(290-420)\end{array}$ & $\begin{array}{l}296 \pm 57 \\
(190-395)\end{array}$ & $\begin{array}{l}311 \pm 36 \\
(250-400)\end{array}$ & $\begin{array}{c}307 \pm 42 \\
(190-420)\end{array}$ \\
\hline $\begin{array}{l}\text { LF mean } \\
\pm \text { S.D. in } \mathrm{mm}\end{array}$ & 1 & $9.9 \pm 0.9$ & $14.4 \pm 2.1$ & $11.4 \pm 3.5$ & $11.9 \pm 2.3$ & $11.8 \pm 2.7$ \\
\hline $\begin{array}{l}\text { HDE mean } \\
\pm \text { S.D. in } \mathrm{mm}\end{array}$ & I & $2.8 \pm 0.6$ & $4.3 \pm 0.9$ & $4.1 \pm 1.1$ & $3.5 \pm 0.8$ & $3.6 \pm 0.9$ \\
\hline $\begin{array}{l}\text { VDE mean } \\
\pm \text { S.D. in mm }\end{array}$ & I & $2.5 \pm 0.6$ & $4.2 \pm 0.8$ & $3.8 \pm 0.8$ & $3.5 \pm 0.7$ & $3.5 \pm 0.9$ \\
\hline Ol mean \pm S.D. & 1 & $2.0 \pm 0.7$ & $4.3 \pm 1.3$ & $4.2 \pm 1.4$ & $3.1 \pm 1.1$ & $3.3 \pm 1.3$ \\
\hline \multirow{2}{*}{$\mathrm{N}$ otoliths } & 1 & 12 & 12 & 14 & 35 & 73 \\
\hline & $r$ & 13 & 13 & 13 & 37 & 76 \\
\hline \multirow{2}{*}{$\begin{array}{l}\text { Length mean } \\
\pm \text { S.D. (min-max) } \\
\text { in } \mathrm{mm}\end{array}$} & I & $\begin{array}{l}1.85 \pm 0.18 \\
(1.57-2.07)\end{array}$ & $\begin{array}{l}2.25 \pm 0.30 \\
(1.80-2.75)\end{array}$ & $\begin{array}{l}2.14 \pm 0.37 \\
(1.27-2.57)\end{array}$ & $\begin{array}{l}2.07 \pm 0.23 \\
(1.72-2.66)\end{array}$ & $\begin{array}{l}2.08 \pm 0.29 \\
(1.27-2.75)\end{array}$ \\
\hline & $r$ & $\begin{array}{l}1.86 \pm 0.19 \\
(1.53-2.11)\end{array}$ & $\begin{array}{l}2.26 \pm 0.31 \\
(1.74-2.68)\end{array}$ & $\begin{array}{l}2.15 \pm 0.38 \\
(1.28-2.61)\end{array}$ & $\begin{array}{l}2.04 \pm 0.24 \\
(1.56-2.60)\end{array}$ & $\begin{array}{l}2.07 \pm 0.29 \\
(1.28-2.68)\end{array}$ \\
\hline \multirow{2}{*}{$\begin{array}{l}\text { Width mean } \\
\pm \text { S.D. (min-max) } \\
\text { in } \mathrm{mm}\end{array}$} & 1 & $\begin{array}{l}1.28 \pm 0.11 \\
(1.09-1.43)\end{array}$ & $\begin{array}{l}1.49 \pm 0.17 \\
(1.27-1.73)\end{array}$ & $\begin{array}{l}1.41 \pm 0.24 \\
(0.99-1.81)\end{array}$ & $\begin{array}{l}1.40 \pm 0.12 \\
(1.20-1.71)\end{array}$ & $\begin{array}{l}1.40 \pm 0.17 \\
(0.99-1.81)\end{array}$ \\
\hline & $r$ & $\begin{array}{l}1.28 \pm 0.11 \\
(1.08-1.42)\end{array}$ & $\begin{array}{l}1.49 \pm 0.17 \\
(1.21-1.73)\end{array}$ & $\begin{array}{l}1.40 \pm 0.23 \\
(1.00-1.77)\end{array}$ & $\begin{array}{l}1.37 \pm 0.13 \\
(1.20-1.79)\end{array}$ & $\begin{array}{l}1.38 \pm 0.17 \\
(1.00-1.79)\end{array}$ \\
\hline \multirow{2}{*}{$\begin{array}{l}\text { Perimeter } \\
\text { mean } \pm \text { S.D. } \\
\text { (min-max) in } \mathrm{mm}\end{array}$} & 1 & $\begin{array}{l}5.10 \pm 0.45 \\
(4.32-5.66)\end{array}$ & $\begin{array}{l}6.10 \pm 0.81 \\
(4.91-7.51)\end{array}$ & $\begin{array}{l}5.92 \pm 1.05 \\
(3.58-7.29)\end{array}$ & $\begin{array}{l}5.62 \pm 0.58 \\
(4.72-7.24)\end{array}$ & $\begin{array}{l}5.67 \pm 0.77 \\
(3.58-7.51)\end{array}$ \\
\hline & r & $\begin{array}{l}5.26 \pm 0.64 \\
(4.19-6.70)\end{array}$ & $\begin{array}{l}6.17 \pm 0.84 \\
(4.84-7.53)\end{array}$ & $\begin{array}{l}5.91 \pm 0.99 \\
(3.65-7.12)\end{array}$ & $\begin{array}{l}5.58 \pm 0.63 \\
(4.38-7.27)\end{array}$ & $\begin{array}{l}5.68 \pm 0.78 \\
(3.65-7.53)\end{array}$ \\
\hline
\end{tabular}


Table II

Kruskal-Wallis and Dunn's tests between morphological and otolithometrical characters of eels caught at different dates. SL, standard length; LF, length of the pectoral fins; HDE, horizontal diameter of the eyes; VDE, vertical diameter of the eyes; OI, ocular index; LO, length of the otoliths; WO, width of the otoliths; PO, perimeter of the otoliths; I, left; r, right; $n . s .$, not significant; ${ }^{*}, P<0.05$; ${ }^{* *}, P<0.01$; ${ }^{* \star *}, \mathrm{P}<0.001 ;{ }^{* * * *}, \mathrm{P}<0.0001$.

Tableau II

Tests de Kruskal-Wallis et de Dunn entre les caractères morphométriques et otolithométriques d'anguilles capturées à différentes dates. SL, longueur standard ; LF, longueur des nageoires pectorales ; HDE, diamètre horizontal des yeux ; VDE, diamètre vertical des yeux ; OI, indice oculaire ; LO, longueur des otolithes ; WO, largeur des otolithes ; PO, périmètre des otolithes ; I, gauche ; r, droit ; n.s., non significatif ; *, $P<0,05$; **, $P<0,01$; **, $P<0,001$; ***, $P<0,0001$.

\begin{tabular}{|c|c|c|c|c|c|c|c|}
\hline & & \multicolumn{2}{|c|}{ K-W test } & \multicolumn{4}{|c|}{ Dunn's test between } \\
\hline \multicolumn{2}{|c|}{ Trait } & $\mathbf{H}$ & p-value & $\begin{array}{c}\text { Mar. 02/ } \\
\text { Oct. } 02\end{array}$ & $\begin{array}{l}\text { Oct. 02/ } \\
\text { Dec. } 02\end{array}$ & $\begin{array}{c}\text { Dec. 02/ } \\
\text { Mar. } 03\end{array}$ & $\begin{array}{c}\text { Mar. 02/ } \\
\text { Mar. } 03\end{array}$ \\
\hline \multicolumn{2}{|l|}{ Age } & 9.3 & * & n.s. & n.s. & n.s. & n.s. \\
\hline \multicolumn{2}{|l|}{ SL } & 16.2 & $\star \star \star *$ & $* \star \star$ & * & n.s. & n.s. \\
\hline LF & I & 21.4 & $* * * *$ & 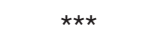 & * & n.s. & * \\
\hline HDE & I & 23.8 & $* * * *$ & $\star * *$ & n.s. & n.s. & n.s. \\
\hline VDE & I & 26.8 & 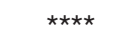 & 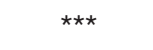 & n.s. & n.s. & $\star *$ \\
\hline Ol & I & 28.3 & $* \star \star \star ~$ & $\star * *$ & n.s. & n.s. & * \\
\hline \multirow{2}{*}{ LO } & I & 11.5 & $\star \star$ & $\star \star$ & n.s. & n.s. & n.s. \\
\hline & $r$ & 12.8 & ** & ** & n.s. & n.s. & n.s. \\
\hline \multirow{2}{*}{ WO } & I & 9.9 & * & * & n.s. & n.s. & n.s. \\
\hline & $r$ & 9.5 & * & * & n.s. & n.s. & n.s. \\
\hline \multirow{2}{*}{ PO } & I & 11.6 & $* *$ & * & n.s. & n.s. & n.s. \\
\hline & $r$ & 10.8 & ** & * & n.s. & n.s. & n.s. \\
\hline
\end{tabular}

Table III

Fluctuating Asymmetry values for three bilateral characters. S.D., standard deviation; (min-max), minimal and maximal values; LO, length of the otoliths; WO, width of the otoliths; PO, perimeter of the otoliths.

Tableau III

Valeurs de l'asymétrie fluctuante de trois caractères bilatéraux. S.D., écart-type ; (min-max), valeurs minimale et maximale; LO, longueur des otolithes; WO, largeur des otolithes; PO, périmètre des otolithes.

\begin{tabular}{lcccccc}
\hline \multicolumn{1}{c}{ Samples } & $\begin{array}{c}\text { March } \\
\text { O2 }\end{array}$ & $\begin{array}{c}\text { October } \\
\mathbf{0 2}\end{array}$ & $\begin{array}{c}\text { December } \\
\mathbf{0 2}\end{array}$ & $\begin{array}{c}\text { March } \\
\mathbf{0 3}\end{array}$ & All \\
\hline \multicolumn{1}{c}{$\mathbf{N}$} & & $\mathbf{1 1}$ & $\mathbf{1 2}$ & $\mathbf{1 3}$ & $\mathbf{3 4}$ & $\mathbf{7 0}$ \\
\hline & LO & $0.02 \pm 0.01$ & $0.03 \pm 0.02$ & $0.01 \pm 0.01$ & $0.03 \pm 0.01$ & $0.02 \pm 0.01$ \\
& & $(0-0.05)$ & $(0-0.06)$ & $(0-0.02)$ & $(0-0.05)$ & $(0-0.06)$ \\
\cline { 2 - 7 } FA & WO & $0.03 \pm 0.02$ & $0.03 \pm 0.01$ & $0.02 \pm 0.02$ & $0.03 \pm 0.03$ & $0.03 \pm 0.02$ \\
Mean \pm S.D. & & $(0.01-0.07)$ & $(0-0.05)$ & $(0.01-0.07)$ & $(0.01-0.09)$ & $(0-0.09)$ \\
(min-max) & PO & $0.04 \pm 0.06$ & $0.02 \pm 0.02$ & $0.02 \pm 0.02$ & $0.03 \pm 0.02$ & $0.03 \pm 0.03$ \\
& & $(0-0.19)$ & $(0-0.07)$ & $(0-0.07)$ & $(0-0.09)$ & $(0-0.19)$ \\
\hline
\end{tabular}




\section{Epidemiological characteristics}

Table IV shows the epidemiological results on 80 yellow eels of Salses-Leucate lagoon. We found a parasite richness of 10 species: 1 Nematoda, 1 Acanthocephala, 1 Monogenea and 7 Digenea. Prevalences of Digenea showed great variations between species, ranging from $1.25 \%$ for species 1 and 2 , to $48.75 \%$ for Lecithochirium gravidum. Digeneans were also characterised by the highest intensities, in particular Prosorhynchus aculeatus (688 parasites in one eel). On the 7 Digenea species, 5 presented a strict specificity for their niche (stomach for the Digenea species 1 and 2 or intestine for the species Prosorhynchus aculeatus, Bucephalus sp. and Deropristis inflata), and 2 were not niche specific, L. gravidum and Helicometra sp.

\section{Table IV}

Epidemiological parameters of the parasite species found in 80 yellow eels Anguilla anguilla at Salses-Leucate lagoon (Pyrénées-Orientales, France). SB, swimbladder; S, stomach; I, intestine; G, gills; Ab. \pm S.D., abundance \pm standard deviation; Prev., prevalence; M. Int. \pm S.D. (min-max), mean intensity \pm standard deviation (minimal and maximal values of individual intensity).

\section{Tableau IV}

Paramètres épidémiologiques relatifs aux espèces parasites rencontrées chez 80 anguilles jaunes Anguilla anguilla provenant de la lagune de Salses-Leucate (Pyrénées-Orientales, France). SB, vessie natatoire; S, estomac; I, intestin; G, branchies; Ab. \pm S.D., abondance \pm écart-type; Prev., prévalence; M. Int. \pm S D. (min-max), intensité moyenne \pm écart-type (valeurs minimale et maximale de l'intensité individuelle).

\begin{tabular}{|c|c|c|c|c|c|}
\hline \multicolumn{2}{|c|}{ PARASITE } & \multirow[t]{2}{*}{ Site } & \multirow{2}{*}{ Ab. \pm S.D. } & \multirow{2}{*}{$\begin{array}{c}\text { Prev. } \\
\%\end{array}$} & \multirow{2}{*}{$\begin{array}{l}\text { M. Int. } \pm \text { S.D. } \\
\text { (min-max) }\end{array}$} \\
\hline Class & Species & & & & \\
\hline Nematoda & Anguillicola crassus & SB & $0.11 \pm 0.45$ & 7.50 & $1.50 \pm 0.84(1-3)$ \\
\hline Acanthocephala & $\begin{array}{l}\text { Acanthocephalus } \\
\text { anguillae }\end{array}$ & I & $0.10 \pm 0.69$ & 3.75 & $2.67 \pm 2.89(1-6)$ \\
\hline Monogenea & Pseudodactylogyrus sp. & $\mathrm{G}$ & 0.04 & 1.25 & 3 \\
\hline \multirow[t]{7}{*}{ Digenea } & $\begin{array}{l}\text { Lecithochirium } \\
\text { gravidum (Hemiuridae) }\end{array}$ & $\begin{array}{c}S \\
1 \\
S+1\end{array}$ & $\begin{array}{l}2.90 \pm 6.01 \\
0.09 \pm 0.36 \\
2.99 \pm 6.09\end{array}$ & $\begin{array}{c}47.50 \\
6.25 \\
48.75 \\
\end{array}$ & $\begin{array}{c}6.11 \pm 7.55(1-35) \\
1.40 \pm 0.55(1-2) \\
6.13 \pm 7.57(1-35)\end{array}$ \\
\hline & $\begin{array}{l}\text { Helicometra sp. } \\
\text { (Opecoelidae) }\end{array}$ & $\begin{array}{c}S \\
1 \\
S+1\end{array}$ & $\begin{array}{l}0.21 \pm 0.90 \\
0.69 \pm 2.08 \\
0.90 \pm 2.50\end{array}$ & $\begin{array}{c}7.50 \\
22.50 \\
26.25 \\
\end{array}$ & $\begin{array}{c}2.83 \pm 1.94(1-6) \\
3.06 \pm 3.54(1-13) \\
3.43 \pm 3.96(1-13)\end{array}$ \\
\hline & $\begin{array}{l}\text { Prosorhynchus } \\
\text { aculeatus } \\
\text { (Bucephalidae) }\end{array}$ & 1 & $20.55 \pm 95.42$ & 23.75 & $\begin{array}{c}86.53 \pm 184.21 \\
(1-688)\end{array}$ \\
\hline & $\begin{array}{l}\text { Bucephalus sp. } \\
\text { (Bucephalidae) }\end{array}$ & 1 & $0.69 \pm 2.14$ & 15.00 & $4.58 \pm 3.68(1-13)$ \\
\hline & $\begin{array}{l}\text { Deropristis inflata } \\
\text { (Acanthocolpidae) }\end{array}$ & I & $2.63 \pm 13.41$ & 16.25 & $\begin{array}{c}16.15 \pm 30.77 \\
(1-109)\end{array}$ \\
\hline & $\begin{array}{l}\text { Digenea species } 1 \\
\text { (Hemiuridae) }\end{array}$ & $S$ & 0.06 & 1.25 & 5 \\
\hline & $\begin{array}{l}\text { Digenea species } 2 \\
\text { (Opecoelidae) }\end{array}$ & $S$ & 0.01 & 1.25 & 1 \\
\hline
\end{tabular}




\section{Relationship between asymmetry and parasitism}

We compared each asymmetrical distance matrice with each epidemiological distance matrice (Table V). The results showed that no significant relationship existed between any of each four FA distance matrices and any of each four epidemiological distance matrices constructed with species belonging to the same class. Moreover, no significant relationship existed between any of each four FA distance matrices and the epidemiological distance matrice constructed with all the parasite species. On the contrary, significant relationships existed between the FA distance matrices constructed with LO and WO and the epidemiological distance matrice constructed with the species found in the stomach (Lecithochirium gravidum, Helicometra sp., Digenea species 1 and 2). However, no significant relationship existed between any of each four FA distance matrice and the epidemiological distance matrice constructed with the species found in the intestine.

\section{Table V}

Mantel tests (1000 iterations) between asymmetrical distance matrices and epidemiological distance matrices. LO, length of the otoliths; WO, width of the otoliths; PO, perimeter of the otoliths; n.s., not significant. Matrices are significantly $(\alpha=0.05)$ connected when $g>1.645$.

\section{Tableau V}

Tests de Mantel (1000 itérations) entre les matrices de distance asymétrique et les matrices de distance épidémiologique. LO, longueur des otolithes ; WO, largeur des otolithes; PO, périmètre des otolithes; n.s., non significatif. Les matrices sont significativement $(\alpha=0,05)$ liées lorsque $g>1,645$.

\begin{tabular}{|c|c|c|c|c|c|c|c|}
\hline \multirow{3}{*}{$\begin{array}{c}\text { Distance } \\
\text { matrices } \\
N=68\end{array}$} & \multicolumn{7}{|c|}{ Parasites } \\
\hline & \multicolumn{4}{|c|}{ Class } & \multicolumn{3}{|c|}{ Site } \\
\hline & Nematoda & $\begin{array}{c}\text { Acantho- } \\
\text { cephala }\end{array}$ & $\begin{array}{l}\text { Mono- } \\
\text { genea }\end{array}$ & Digenea & $\begin{array}{c}\text { All } \\
\text { species }\end{array}$ & Stomach & Intestine \\
\hline LO & n.s. & n.s. & n.s. & n.s. & n.s. & $\begin{array}{l}Z=494 \\
g=2.51\end{array}$ & n.s. \\
\hline WO & n.s. & n.s. & n.s. & n.s. & n.s. & $\begin{array}{l}Z=494 \\
g=2.51\end{array}$ & n.s. \\
\hline $\mathrm{PO}$ & n.s. & n.s. & n.s. & n.s. & n.s. & n.s. & n.s. \\
\hline All traits & n.s. & n.s. & n.s. & n.s. & n.s. & n.s. & n.s. \\
\hline
\end{tabular}

\section{DISCUSSION}

\section{Morphological and otolithometrical characteristics}

Major morphological and otolithometrical differences were found between eels caught in March 02 and eels caught in October 02. These results may be related to the difference in the growth rate of organisms between seasons. Growth rate of eels depends on two major factors, diet and temperature (BERG, 1989), which both present typical seasonal variations, since they increase during summer. Morphological differences were also found between eels caught in March 02 and eels caught in March 03, which suggests that the morphological structure of yellow eels in a same location could be strongly influenced by environmental factors presenting inter-annual variations. 
We quantified an average asymmetry ranging from 1 to $4 \%$ for the three bilateral traits among the yellow eel sample of Salses-Leucate lagoon, which is close to what has been found in other fish species (SASAL and PAMPOULIE, 2000; LOOT et al., 2001). The fact that asymmetry showed a rather homogeneous pattern from date to date may be related to a rather persistence of abiotic and biotic stresses associated to a specific environment and/or a homogenous pattern of FA for a given population in a given place. The difference of asymmetry of the length of otoliths recorded between two successive sampling dates is more difficult to discuss. However, otoliths constitute some permanent recorders of the exposure to environment (CAMPANA et al., 1997), since they are characterised by a daily growth (PANNELLA, 1971). Their size variation could be related to many factors that could differ consequently from day to day (access to food, local water conditions...). The traits showed generally heterogeneous patterns of FA, that means a low concordance between FA of traits in an individual (NOSIL and REIMCHEN, 2001; PÉLABON et al., 2004). That is in accordance with our result. It has been suggested that the different traits of an organism would not be equal towards asymmetry. Such a trait-specific susceptibility has been explained by different levels of developmental stability or could depend on the amount of energy and structural components needed to form a unit of length of a trait, i. e. the cost of growth of a trait (APARICIO and BONAL, 2002). The absence of relationship between the asymmetry of length and the asymmetry of width of otoliths seems to show that such a difference of susceptibility could also exist between traits within a same functional unit.

\section{Epidemiological characteristics}

Comparisons of epidemiological values with other previously published data are delicate because of differences in the sampling protocol, sample size and host size/age (THOMAS and OLLEVIER, 1992; MOLNÁR et al., 1994; ROHDE, 1994; POULIN, 2000), but also because of potential seasonal variations and differences in habitat characteristics (CONNEELY and MCCARTHY, 1986). We thus decided to minimize the sources of variation by considering epidemiological characteristics of eels (i) having the same size range and (ii) coming from brackish waters (Table VI). In terms of parasite richness, our results (10 species) concurred with those found in Italy (10 species, KENNEDY et al., 1997) or in Spain (11 species, MAILLO et al., 2000). The prevalence of the species occurring in all stations is clearly different. The prevalence of the monogenean species Pseudodactylogyrus $\mathrm{sp}$. from Salses-Leucate lagoon is the lowest and presents a great variation from site to site. The digenean species are also characterised by a great variability in the prevalence values. This could be related to a seasonal variation in the life cycle and/or a local absence of one intermediate host. The comparison between sites where $A$. crassus is present shows that the nematode has the lowest prevalence in eels of Salses-Leucate lagoon. Eels at Salses-Leucate lagoon may be less infected due to the relatively high salinity of the water - usually $33 \%$ (RSL, 2005). Actually, a negative relationship between salinity and $A$. crassus infestation level is commonly reported: parasite development and transmission appears to be lower in brackish and marine waters than in freshwaters (KIRK et al., 2000; SAUVAGET et al., 2003). The weak presence of the nematode in Salses-Leucate could also be due to the mortality of heavily infected eels (GORDON and RAU, 1982; FALIEX and MORAND, 1994; THOMAS, 2002).

\section{Parasitism and asymmetry}

Relationship between asymmetry and parasitism has been studied in several fish models (BERG et al., 1997; BERGSTROM and REIMCHEN, 2000; SASAL and PAMPOULIE, 2000; LOOT et al., 2001; NOSIL and REIMCHEN, 2001). However, results were not systematically convincing because (i) the measured traits could response differently to the presence of the parasites and (ii) parasite species or classes may have different effects on the development of the measured traits. In order to integrate all these possibilities, we decided to consider (i) each measured trait and each parasite class/niche 


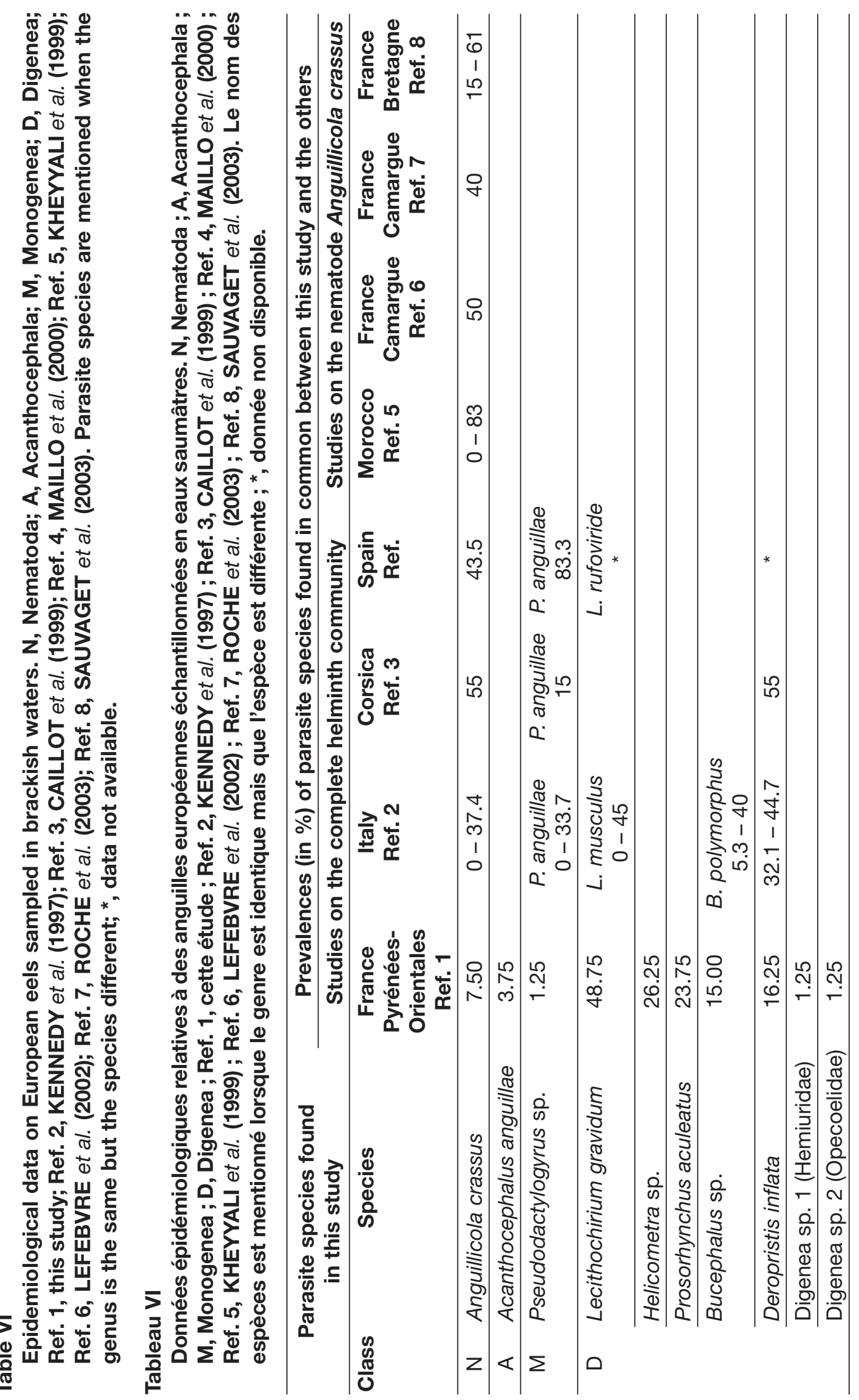


independently and (ii) to confront all the measured traits and all the parasite species in two distance matrices.

We found no relationship between asymmetry and parasitism, excepted for parasites found in the stomach. Our results suggest that neither the different classes of parasites nor the whole community represents causes or consequences of the yellow eel asymmetry.

Our result may suggest that no ecological difference, in terms of diet composition and exposure to infectious agents, exist between asymmetric and symmetric eels. Parasites found in the studied eels were both direct (monogeneans) and complex life-cycle parasites (digeneans, nematodes and acanthocephalans). In the last case, eels acquired the parasite by eating infected intermediate hosts. The absence of difference in parasite community composition between asymmetric and symmetric eels may reveal that there is no difference in space occupation or prey choice between the studied eels. Thereafter, we can hypothesise that other factors are responsible of the observed asymmetries.

The genetic incapacity of the fish to buffer developmental anomalies (asymmetry) has been supposed to be related with the susceptibility to parasitism (MØLLER, 1996). That means that the presence of parasites could be a consequence of the asymmetry and not the cause. The yellow eel is the final host for all the parasite species found. In this study, because the parasite species found have to reproduce in the eel, i.e. the final host, we may assume that the fitness of the parasite is strongly linked with the one of the host. Selective pressures should thus have favoured the lowest virulence of the parasites and the highest resistance of the eels and this should be even truer for ancient host parasite interactions. Pseudodactylogyrus sp. and Anguillicola crassus constitute younger eel parasite associations as they have been introduced in the last 30 years (BRUSLÉ, 1994). These parasite species should thus be characterized by a higher virulence (COMBES, 1995), which is confirmed by some massive mortalities induced by $A$. crassus (MOLNÁR et al., 1991; BARUŠ and MORAVEC, 1999). We may hypothesise that they should be better tags for asymmetric eels. However, our results seem not to support this hypothesis, as we did not find any relationship between these invasive species and asymmetry.

Our result seems to be in agreement with the fact that parasite community as a whole could not be considered as a cause of the yellow eel asymmetry, because of a suspected low metabolic cost. However, a positive relationship between the asymmetry of length and width of otoliths and parasites found in the stomach (Lecithochirium gravidum, Helicometra sp., digenean species 1 and 2) was established. Even if the precise pathway by which the digeneans exert their influence on the eel remains undocumented, by definition parasites remove a fraction of the available energy for the host. Moreover, digenean infections may induce general and local physiological disorders in the gastrointestinal tract. On the one hand, several studies on gastrointestinal parasitism, concerning a wide range of parasitehost systems, have shown a chronic reduction in food intake and a lost of the digestive and absorptive capacities of the mucosa at the infection site (HOSTE, 2001, for a review). On the other hand, the stomach of fishes presents absorptive capacities (BUDDINGTON and DIAMOND, 1987). Digeneans could thus be responsible for a part of the asymmetry by disturbing directly or indirectly some nutrients metabolism. Otolith measurements may rapidly vary and therefore may be more prone to translate stresses caused by parasites into asymmetry. Current work on the direct physiological effects of the parasites on the eels will be published shortly.

\section{ACKNOWLEDGEMENTS}

The authors would like to thank Mr ESTIRACH and CYPRIEN for supplying with eels. This research was supported by the "Centre National de la Recherche Scientifique" (CNRS), the "Université de Perpignan" (UP), the "GRoupe d'Intérêt Sur les poissons AMphihalins" (GRISAM) and the "Observatoire Régional Méditerranéen sur l'Environnement" (ORME). 


\section{REFERENCES}

ALIBERT P., BOLLACHE L., CORBERANT D., GUESDON V., CÉZILLY F., 2002. Parasitic infection and developmental stability: fluctuating asymmetry in Gammarus pulex infected with two acanthocephalan species. J. Parasitol., 88, 47-54.

ALTIZER S., HARVELL D., FRIEDLE E., 2003. Rapid evolutionary dynamics and disease threats to biodiversity. Trends Ecol. Evol., 18, 589-596.

APARICIO J.M., BONAL R., 2002. Why do some traits show higher fluctuating asymmetry than others? A test of hypotheses with tail feathers of birds. Heredity, 89, 139144.

BARUŠ V., MORAVEC F., 1999. Anguillicolosis of the European eel (Anguilla anguilla) in the Czech Republic. Czech. J. Anim. Sci., 44, 423-431.

BERG R., 1989. The growth of eels: a critical assessment of data from open waters. EIFAC Working Party on Eel, Porto, 11 p.

BERG O.K., ADKISON M.D., QUINN T.P., 1997. Bilateral asymmetry, sexual dimorphism, and nematode parasites in mature male Sockeye salmon Oncorhynchus nerka. Northwest Sci., 71, 305-312.

BERGSTROM C.A., REIMCHEN T.E., 2000. Functional implications of fluctuating asymmetry among endemic populations of Gasterosteus aculeatus. Behaviour, 137, 1097-1112.

BLACKER R.W., 1974. Recent advances in otolith studies. In: Sea fisheries research. Wiley, New-York, USA, pp. 67-90.

BROWN C.R., BROWN M.B., 2002. Ectoparasites cause increased bilateral asymmetry of naturally selected traits in a colonial bird. J. Evol. Biol., 15, 1067-1075.

BRUSLÉ J., 1990. L'anguille européenne (Anguilla anguilla), une espèce jugée commune jusqu'à la dernière décennie, mérite-t-elle d'acquérir aujourd'hui le statut d'espèce menacée? Bull. Soc. Zool. Fr., 114, 61-73.

BRUSLÉ J., 1994. L'anguille européenne Anguilla anguilla, un poisson sensible aux stress environnementaux et vulnérable à diverses atteintes pathogènes. Bull. Fr. Pêche Piscic., 335, 237-260.

BUDDINGTON R.K., DIAMOND J.M., 1987. Pyloric ceca of fish: a new absorptive organ. Am. J. Physiol., 252, 65-76.

BUSH A.O., LAFFERTY K.D., LOTZ J.M., SHOSTAK A.W., 1997. Parasitology meets ecology on its own terms: Margolis et al. revisited. J. Parasitol., 83, 575-583.

CAILLOT C., MORAND S., MÜLLER-GRAF C.M., FALIEX E., MARCHAND B., 1999. Parasites of Dicentrarchus labrax, Anguilla anguilla, and Mugil cephalus from a Pond in Corsica, France. J. Helminthol. Soc. Wash., 66, 95-98.

CAMPANA S.E., THORROLD S.R., JONES C.M., GUNTHER D., TUBRETT M., LONGERICH H., JACKSON S., HALDEN N.M., KALISH J.M., PICCOLI P., PONTUAL H.D., TROADEC H., PANFILI J., SECOR D.H., SEVERIN K.P., SIE S.H., THRESHER R., TEESDALE W.J., CAMPBELL J.L., 1997. Comparison of accuracy, precision and sensitivity in elemental assays of fish otoliths using the electron microprobe, proton-induced X-ray emission, and laser ablation inductively coupled plasma mass spectrometry. Can. J. Fish. Aquat. Sci., 54, 2068-2079.

CASTELNAUD G., ROCHARD E., 1994. Surveillance halieutique de l'estuaire de la Gironde. Suivi statistique 1992, étude de la faune circulante 1993. CEMAGREF de Bordeaux/EDF, $155 \mathrm{p}$. 
CLARKE G.M., 1998. Developmental stability and fitness: the evidence is not quite so clear. Am. Nat., 152, 762-766.

COMBES C., 1995. Interactions durables: écologie et évolution du parasitisme. Masson, Paris, $524 \mathrm{p}$.

CONNEELY J.J., MCCARTHY T.K., 1986. Ecological factors influencing the composition of the parasite fauna of the European eel, Anguilla anguilla (L.), in Ireland. J. Fish Biol., 28, 207-219.

DASZAK P., CUNNINGHAM A.A., HYATT A.D., 2000. Emerging infectious diseases of wildlife. Threats to biodiversity and human health. Science, 21, 443-449.

DAVID P., HINGLE A., FOWLER K., POMIANKOWSKI A., 1999. Measurement bias and fluctuating asymmetry estimates. Anim. Behav., 57, 251-253.

ELIE P., ROCHARD E., BABIN D., 1994. Acquisition de données par pêche en vue de la gestion de populations de poissons - contraintes et méthodes en fonction des objectifs: exemple de l'anguille. Colloque Franco-Québecois sur l'intégration des technologies modernes à la gestion des poissons dulcicoles et amphihalins. Québec, juin 1992. Ministère de l'Environnement et de la Faune, Service de la Faune Aquatique, $210 \mathrm{p}$.

FALIEX E., MORAND S., 1994. Population dynamics of the metacercarial stage of the bucephalid trematode, Labratrema minimus (Stossich, 1887) from Salses-Leucate lagoon (France) during the cercarial shedding period. J. Helminthol., 68, 35-40.

GORDON D.M., RAU M.E., 1982. Possible evidence for mortality induced by the parasite Apatemon gracilis in a population of brook sticklebacks (Culaea inconstans). Parasitology, 84, 41-47.

HOSTE H., 2001. Adaptive physiological processes in the host during gastrointestinal parasitism. Int. J. Parasitol., 31, 231-244.

KENNEDY C.R., DI CAVE D., BERRILLI F., ORECCHIA P., 1997. Composition and structure of helminth communities in eels Anguilla anguilla from Italian coastal lagoons. J. Helminthol., 71, 35-40.

KHEYYALI D., LACHHEB K., YAHYAOUI A., HOSSAINI-HILALI J., 1999. Status of European eel infestation by the nematode Anguillicola crassus in aquatic ecosystems in Morocco. Actes Inst. Agron. Vet. (Maroc), 19, 177-180.

KIRK R.S., KENNEDY C.R., LEWIS J.W., 2000. Effect of salinity on hatching, survival and infectivity of Anguillicola crassus (Nematoda: Dracunculoidea) larvae. Dis. Aquat. Organ., 40, 211-218.

KOSKIVAARA M., VALTONEN E.T., 1992. Dactylogyrus (Monogenea) communities on the gills of roach in three lakes in Central Finland. Parasitology, 104, 263-272.

LECOMTE-FINIGER R., 1985. L'âge de l'anguille européenne (Anguilla anguilla). J. Appl. Ichthyol., 4, 178-192.

LEFEBVRE F., CONTOURNET P., PRIOUR F., SOULAS O., CRIVELLI A.J., 2002. Spatial and temporal variation in Anguillicola crassus counts: results of a 4 year survey of eels in Mediterranean lagoons. Dis. Aquat. Organ., 50, 181-188.

LOOT G., LEK S., BROWN S.P., GUEGAN J.F., 2001. Phenotypic modification of roach (Rutilus rutilus L.) infected with Ligula intestinalis L. (Cestoda: Pseudophyllidea). J. Parasitol., 87, 1002-1010.

MAILLO P.A., SALVADO H., GRACIA M.P., MARQUES A., 2000. Parasite fauna on the European eel, Anguilla anguilla, L. 1758, in the natural park of the Ebro delta, West 
Mediterranean. $38^{\mathrm{e}}$ meeting annuel du groupement des protistologues de langue française, Paris, France.

MANTEL N., 1967. The detection of disease clustering and generalized regression approach. Cancer Res., 27, 209-220.

MARKOV T.A., 1995. Evolutionary ecology and developmental stability. Annu. Rev. Entomol., 40, 105-120.

MØLLER A.P., 1992. Parasites differentially increase the degree of fluctuating asymmetry in secondary sexual characters. J. Evol. Biol., 5, 691-699.

MØLLER A.P., 1996. Parasitism and developmental instability in host: a review. Oikos, 397-405.

MØLLER A.P., SWADDLE J.P., 1997. Asymmetry, Developmental Stability, and Evolution. Oxford University Press, $291 \mathrm{p}$.

MOLNÁR K., SZEKELY C.S., BASKA F., 1991. Mass mortality of eel in Lake Balaton due to Anguillicola infection. Bull. Eur. Assoc. Fish Pathol., 11, 211-212

MOLNÁR K., SZEKELY C.S., PERÉNYI M., 1994. Dynamics of Anguillicola crassus (Nematoda: Dracunculoidea) infection in eels of Lake Balaton, Hungary. Folia Parasitol., 41, 193-202.

MORIARTY C., 1983. Age determination and growth rate of eels, Anguilla anguilla. J. Fish Biol., 23, 257-264.

MORIARTY C., 1987. Factors infuencing recruitment of the Atlantic species of anguillid eels. Am. Fish. Soc. Symp., 1, 483-491.

NOSIL P., REIMCHEN T.E., 2001. Tarsal asymmetry, nutritional condition, and survival in water boatmen (Callicorixa vulnerata). Evolution, 55, 712-720.

PALMER A.R., 1999. Detecting publication bias in meta-analyses: a case study of fluctuating asymmetry and sexual selection. Am. Nat., 154, 220-233.

PALMER A.R., STROBECK C., 1986. Fluctuating asymmetry: measurement, analysis, patterns. Ann. Rev. Ecol. Syst., 17, 391-421.

PANKHURST N.W., 1982. Relation of visual changes to the onset of sexual maturation in the European eel Anguilla anguilla (L.). J. Fish Biol., 21, 127-140.

PANNELLA G., 1971. Fish otoliths: daily growth layers and periodical patterns. Science, $173,1124-1127$.

PÉLABON C., HANSEN T.F., CARLSON M.L., ARMBRUSTER W.S., 2004. Variational and genetic properties of developmental stability in Dalechampia scandens. Evolution, 58, 504-514.

POLAK M., 1993. Parasites increase fluctuating asymmetry of male Drosophila nigrospiracula: implication for sexual selection. Genetica, 89, 255-265.

POULIN R., 2000. Variation in the intraspecific relationship between fish length and intensity of parasitic infection: biological and statistical causes. J. Fish Biol., 56, 123-137.

REIMCHEN T.E., 1997. Parasitism of asymmetrical pelvic phenotypes in stickleback. Can. J. Zool., 75, 2084-2094.

ROCHE H., BUET A., RAMADE F., 2003. Caractéristiques écophysiologiques d'une population d'anguilles de Camargue exposée à une pollution clandestine par des polluants organiques persistants. Rev. Ecol. (Terre Vie), 58, 103-125. 
ROHDE K., 1994. Niche restriction in parasites: proximate and ultimate causes. Parasitology, 109, 69-84.

RSL, 2005. Réseau de suivi lagunaire. IFREMER Sète. www.ifremer.fr/delst/surveillance/ index.htm.

SASAL P., PAMPOULIE C., 2000. Asymmetry, reproductive success and parasitism of Pomatoschistus microps in a French lagoon. J. Fish Biol., 57, 382-390.

SASAL P., DESDEVISES Y., DURIEUX E., LENFANT P., ROMANS P., 2004. Parasites in marine protected areas: success and specificity of monogeneans. J. Fish Biol., 64, 370-379.

SAUVAGET B., FATIN D., BRIAND C., 2003. Contamination par Anguillicola crassus de cinq populations d'anguilles (Anguilla anguilla) du littoral de Bretagne sud (France). Bull. Fr. Pêche Piscic., 368, 21-26.

THOMAS J.D., 2002. The ecology of fish parasites with particular reference to helminth parasites and their salmonid fish hosts in Welsh rivers: a review of some of the central questions. Adv. Parasitol., 52, 1-154.

THOMAS K., OLLEVIER F., 1992. Paratenic hosts of the swimbladder nematode Anguillicola crassus. Dis. Aquat. Organ., 13, 165-174.

THOMAS F., WARD D.F., POULIN R., 1998. Fluctuating asymmetry in an insect host: a big role for big parasites? Ecol. Lett., 1, 112-117

VAN VALEN L., 1962. A study of fluctuating asymmetry. Evolution, 16, 125-142.

WIRTH T., BERNATCHEZ L., 2003. Decline of North Atlantic eels: a fatal synergy? Proc. $R$. Soc. Lond. B. Biol. Sci., 270, 681-688. 
